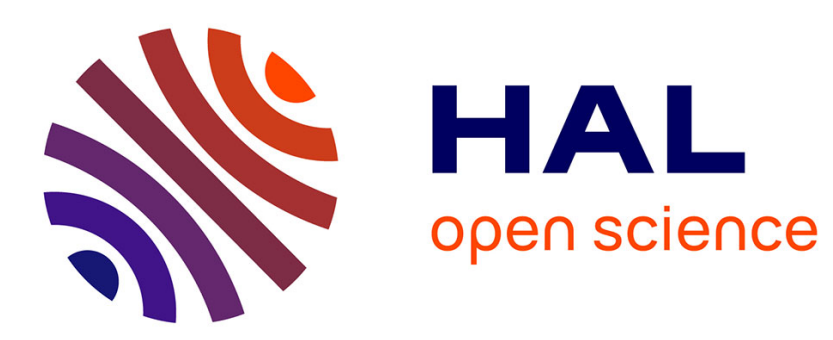

\title{
MyOwnLife: incremental and hierarchical classification of a personal image collection on mobile devices
}

\author{
Antoine Pigeau
}

\section{To cite this version:}

Antoine Pigeau. MyOwnLife: incremental and hierarchical classification of a personal image collection on mobile devices. IEEE Conf. on Multimedia and Expo (ICME'2008), Jun 2008, Hannover, Germany. pp.873 - 876. hal-00463825

\section{HAL Id: hal-00463825 \\ https://hal.science/hal-00463825}

Submitted on 15 Mar 2010

HAL is a multi-disciplinary open access archive for the deposit and dissemination of scientific research documents, whether they are published or not. The documents may come from teaching and research institutions in France or abroad, or from public or private research centers.
L'archive ouverte pluridisciplinaire HAL, est destinée au dépôt et à la diffusion de documents scientifiques de niveau recherche, publiés ou non, émanant des établissements d'enseignement et de recherche français ou étrangers, des laboratoires publics ou privés. 


\title{
MYOWNLIFE: INCREMENTAL SUMMARIZATION OF A PERSONAL IMAGE COLLECTION ON MOBILE DEVICES
}

\author{
Antoine Pigeau \\ LINA (CNRS FRE 2729), INRIA ATLAS group \\ 2, rue de la Houssiniére 44322 Nantes cedex 03 - France \\ firstname.lastname@univ-nantes.fr
}

\begin{abstract}
In this paper, we propose a new method to summarize a personal image collection, to provide a structure adapted to interface constraints on mobile devices. An incremental hierarchical algorithm and a method of textual representation of each obtained event are proposed to build a geo-temporal classification. Results are validated with our prototype MyOwnLife on real data sets.
\end{abstract}

Index Terms - Personal image classification, mobile device, hierarchical clustering

\section{Goal and existing works}

Building of personal multimedia collection is now widespread thanks to common mobile devices equipped with digital camera. Providing solutions to browse such a collection is then a research area of much interest, requiring to deal with a large amount of data of different kind (audio, video and textual). Moreover, the difficulty of the task is increased on mobile devices due to their interface constraints. In this paper, we focus on personal image collections, which present the advantage on common image collections to have rich meta data, as time stamp and geographical information (from GPS system). The objective of our work is to provide summaries of the collection in order to facilitate the browsing task on a mobile device, a pertinent device to share/browse personal images due to its continuous availability.

The main steps of our approach are:

1.incremental building of a hierarchical temporal classification: we propose an improvement of our incremental and hierarchical algorithm [1], which provides a classification from the time stamp of each image. With our approach, we tend to emphasize the browsing task, rather than querying (a point motivated by the partial memory that the user has of the collection).

2.textual representation of obtained classes: each obtained class at the previous step is represented with a textual set of labels. The goal is to provide a succinct and simple representation of each event. Those summaries are built from new meta-data obtained from initial time stamp and Geographical Information System (GIS). The advantage of such approach are the energy saving, since image displaying is costly.

3.combination of temporal and geographical information: to improve our hierarchical temporal classification, we propose a method to re-structure classes based on their geographical information. Our approach consists in merging successive temporal nodes with similar geographical summary (similar location based on a geographical map).

For existing automated schemes at research stage, time stamp has long been a favourite since it is an intuitively appealing, cheap and reliable measurement. Segmenting the sequence of time stamps has been viewed in $[2,3]$ as the incremental detection of gaps. Some thresholding sets the definition of a "meaningful" gap. But those solutions do not seem to provide hierarchical partitions in an unsupervised manner.

The work closest to the present paper is [4], which also organizes an image collection hierarchically, based on time and location clusters. To our understanding, their work incorporates a series of rules derived from user's expectations to build a geo-temporal hierarchy of events. Contrary to our work the number of levels in the hierarchy is set manually. Furthermore, their scheme is not incremental, but works in batch mode. In our view, incrementality is necessary to keep the collection organized without user help.

The remainder of this paper is organized as follows. Section 2 discloses the process to track a temporal hierarchy. Section 3 provides the building of a geo-temporal hierarchy. Finally, our approach is validated with experiments and the work is summarized in Section 4.

\section{Temporal hierarchical partition}

We opt here for a combination of a supervised clustering, for the finer partition, and the mixture model framework, for the coarser partitions (providing several summaries of the finer partition). As in [4], we propose to set manually the precision degree of the finer partition fixing manually a boundary between events.Such solution seems meaningful since it corresponds to the building process of a personal image collection [4], and provides a clear and robust initial partition to build the summaries. Each leave of our temporal tree is then an event with temporally connected images.

Based on the obtained finer partition, we then propose to 


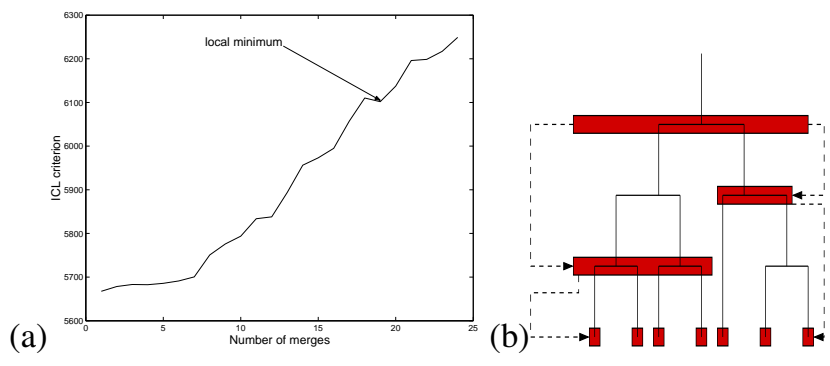

Fig. 1. Selection of levels corresponding to local optima of the ICL criterion: (a) the optimal ICL criterion found at each level of the binary tree represented on (b) is plotted. The grey rectangles indicate the corresponding selection of partitions. Once an optimum is found at a level $q$, we search for another local optima in each subtree from $q$. 'Local' minima here is to be interpreted as follows: both slightly coarser and slightly finer partitions are worse, in the ICL sense.

build a hierarchical classification to provide several coarser summaries. Our approach is based on a mixture model framework. The main advantages is the availability of probabilistic criteria to select model complexities. The building of the summaries consists then in the following steps: (1) retrieve Gaussian model parameters from the finer partition (one Gaussian component per initial classes), (2) apply a hierarchical algorithm [5], to provide a binary tree from a Gaussian model, and (3) select pertinent levels thanks to the Integrated Likelihood Criterion (ICL)[6], to avoid uninteresting and strongly redundant partitions. The ICL criterion provides a consistent solution to the issue.

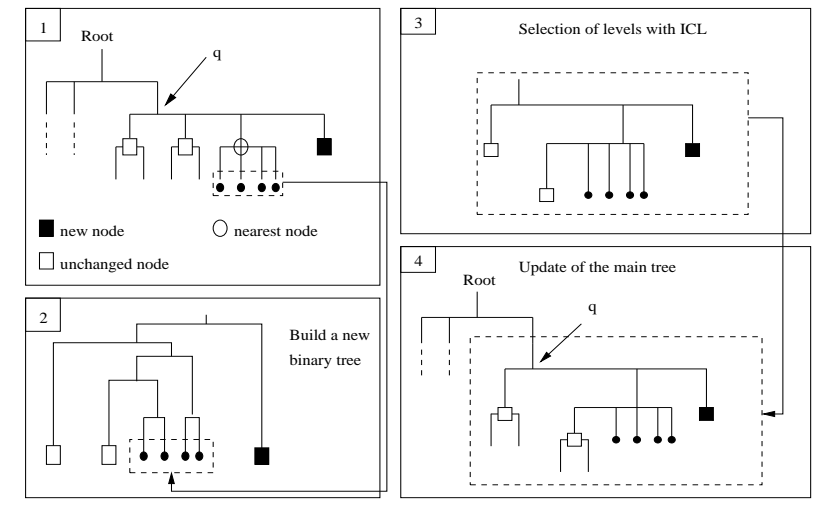

Fig. 2. Update of a subtree: We add the new data group by the root, retrieve the model associated to its children and compute the likelihood of the new group based on the initial parameters. In this example, the new group is affected to the component $q$ (the likelihood is higher than $\beta$ and the choice of the node $q$ is based on the MAP criterion). The update is then propagated to the node $q$ : we retrieve the model associated to its children and compute the likelihood of the new group. Here we make the hypothesis that it is lower than $\beta$, we then update the subtree of root $q$. Fig. 2.1 presents the new node associated to the new group, its nearest node (here, the parameter $N_{\text {near }}=1$ ) and the unchanged nodes. We then re-build a binary tree from the unchanged node, the leaves of the nearest node and the new node (fig. 2.2) and select the relevant levels based on the ICL criterion. Fig. 2.3 presents the obtained new subtree after our ICL selection. Finally the main initial tree is updated with the subtree (fig. 2.4).
As the process is incremental, the first step of our algorithm is to group together new images with less than $T_{\text {diff }}$ hours of time differences. Each group is then added by the root of our hierarchical classification and this node $q$ is updated as follows:

1. If $q$ is the root without any child, the group is added as a new child of $q$;

2. if $q$ is a leaf, we add the new group in this node and the parameters of the tree are updated (from $q$ to the root).

3. else:

3.1. detect the change due to the new image group: we retrieve the model composed of $q$ 's children and compute the likelihood $l$ of the new group based on the obtained parameter of the Gaussian model. If $l>\beta$, it means that the new group is associated with an existing cluster (selected with the MAP criterion) and we update this node (and re-iterate step 1); else we add a new component $q_{\text {new }}$, a new child of $q$, associated with the new image group and go to the next step;

3.2. search for summaries: we build a new binary tree from the leaves of the $N_{\text {near }}$ nearest nodes of $q_{\text {new }}$ and the others children of $q$ with the algorithm [5]. This agglomerative algorithm provides a binary tree from a Gaussian Mixture;

3.3. select the summaries in the obtained binary tree. This selection is carried out with the ICL criterion (fig.1);

3.4. merge the updated subtree with the initial one.

Step 3(1) checks if the initial parameters fit its associated data including the new image group. The decision to add the new group in the current level or to propagate the update depend on the threshold $\beta$. A high likelihood for the new data involves that the parameters fit them well. The value of $\beta$ is set manually: the higher $\beta$, the more distinct are the classes in the summaries (but the calculation complexity is increased). The selection of the $N_{\text {near }}$ neighbours at step 3(2) attempt to avoid poor summaries, due to the incremental property of our algorithm. Indeed a new group of images can have an influence on the whole tree (it could lead to a new coarser summary). The parameter $N_{\text {near }}$ enables then to set the number of neighbours from which the search for new summary is carried out from the leaves (others nodes on the same updated level are also used but not from their leaves). The higher $N_{\text {near }}$, the higher are the quality of summaries and the calculation complexity. Figure 2 presents the different steps of our algorithm.

\section{Geo-temporal partition}

We present here a method to summarize a class with textual labels and then a technique to improve our temporal classification, combining it with geographical textual summaries.

Let us recall that the initial meta-data recorded with the mobile for each image are the time stamp and the location. Then a knowledge base can be used to provide usermeaningful information from the initial meta-data. Given a GPS coordinate, a GIS (Geographical Information System) 


\begin{tabular}{|}
\begin{tabular}{|c|c|c|c|c|c|}
\hline id & decade & year & season & month & $\pi$ \\
\hline 1 & 90 's & 1999 & winter & December & 1.0 \\
\hline 2 & 00 's & 2000 & winter & January & 0.9 \\
\hline 3 & 00 's & 2000 & winter & January & 1.0 \\
\hline id & \multicolumn{6}{|c|}{ continent } & country & state & city \\
\hline 1 & North America & USA & New York & Rochester \\
\hline 2 & North America & USA & New York & NY city \\
\hline 3 & North America & USA & New York & NY city \\
\hline
\end{tabular}
\end{tabular}

Table 1. Example of attributes and values obtained respectively from the time stamp and the location.

could provide the continent, the country and so forth. Tables 1 present obtained temporal and geographical meta-data for 3 images, defined on 4 attributes in both cases. Notice that the attributes are organized in a hierarchical way.

First, an image $i$ is defined by 2 temporal and geographical sets of meta-data:

$$
\begin{aligned}
m_{i}= & \left\{\left\langle t_{1}, t_{2}, \ldots, t_{L} \mid t_{l} \in M_{t}\right\rangle,\right. \\
& \left.\left\langle s_{1}, s_{2}, \ldots, s_{L^{\prime}} \mid s_{l} \in M_{s}\right\rangle\right\}
\end{aligned}
$$

where $t_{l}$ (or $s_{l}$ ) is a textual label defined for the attribute $l$, $L$ (resp. $L^{\prime}$ ) is the number of temporal (resp. geographical) attributes to represent an image, according to the knowledge base, and $M_{t}$ and $M_{s}$ are respectively the sets of possible label values defined on temporal and geographical attributes.

Then, we build the class summary with the textual sets associated to its contained images. Let $k$ be a class, its summary is defined as:

$$
\begin{aligned}
c_{k}= & \left\{\left\langle t_{1}, \ldots, t_{l-1},\left\{\alpha_{1} / t_{l}^{1}, \ldots, \alpha_{r} / t_{l}^{r}\right\}\right\rangle,\right. \\
& \left.\left\langle s_{1}, \ldots, s_{l^{\prime}-1},\left\{\alpha_{1^{\prime}} / s_{l^{\prime}}^{1}, \ldots, \alpha_{r^{\prime}} / s_{l^{\prime}}^{r^{\prime}}\right\}\right\rangle\right\},
\end{aligned}
$$

where $t_{l} \in M_{t}, s_{l^{\prime}} \in M_{s}, l$ and $l^{\prime}$ are the first attributes from which label values present differences. For the attribute $l$ (or $l^{\prime}$ ), the summary is represented by $r$ (or $r^{\prime}$ ) different values associated to the contained images: $\alpha$ is the average weight of each textual label. This score, for both the temporal and spatial case, is the average of the image-to-class assignment $\pi$ (see temporal Table 1 ) for each textual value:

$$
\alpha_{t_{l}^{r}}=\frac{\sum_{i \mid t_{r}^{l}=t_{i}} \pi_{i}}{\sum_{i=1}^{n_{k}} \pi_{i}}
$$

where $\pi_{i}$ is the assignment probability of the image $i$ to its class and $n_{k}$ is the number of images in the class. Such an approach enables to emphasize labels strongly associated to its class, labels which are likely to better represent its content. For example, if a class contains the images of Tables 1, its class summary is defined as:

$$
c_{1}=\left\{\left\langle\left\{\frac{1}{2.9}=0.35 / \mathbf{9 0} \text { 's, } \frac{1.9}{2.9}=0.65 / \mathbf{0 0} \mathbf{s}\right\}\right\rangle,\right.
$$

$\langle$ North A., USA, NY, $\{0.35 /$ Rochester, $0.65 /$ NY city $\}\rangle\}$
Here we have $l=1$ and $l^{\prime}=3$. The attributes Years and Cities present different values.Stopping the textual summary at attributes with different values enables to simplify their visual representation and avoid building sets of labels that do not belong to the same time interval or location.

The same principle is applied to obtain the textual summaries of a node's children, called level summaries. First we build the class summary of each child and then detect the attribute which presents different values among each summary. Each child is then only represented with the attributes with similar values until the first attribute with different values. For example, let $c_{2}$ a class summary defined as:

$$
c_{2}=\{\langle\mathbf{0 0} \text { 's, }\{0.42 / \mathbf{2 0 0 5}, 0.58 / \mathbf{2 0 0 6}\}\rangle
$$

\section{$\langle$ Europe, France, Île de F., $\{0.33 /$ Paris, $0.66 /$ NY city $\}\rangle\}$}

And let $c_{1}$ and $c_{2}$ two children of a node $c$, its level summaries is then defined as:

$$
\begin{aligned}
& c_{\text {children }\left(c_{1}\right)}=\{\langle\{0.33 / \mathbf{9 0} \text { 's }, 0.66 / \mathbf{0 0} \text { 's }\}\rangle,\langle\text { North America }\rangle\} \\
& c_{\text {children }\left(c_{2}\right)}=\{\langle\mathbf{0 0} \text { 's }\rangle,\langle\text { Europe }\rangle\}
\end{aligned}
$$

The summaries are limited to the Decade and Continent attributes. It enables to emphasize the main differences between events on a same level.

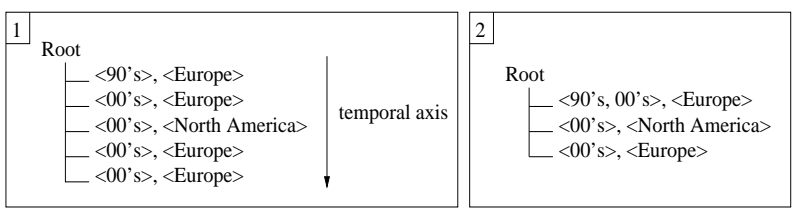

Fig. 3. Figure 3.1 represents the initial temporal classification where each node is defined with its level-summary. Geo-temporal partition is then obtained by merging temporal continuous nodes with similar geographical summary, as showed on fig. 3.2. Note that the merge step includes the update of the temporal summary of each obtained hybrid node (first node on fig. 3.2).

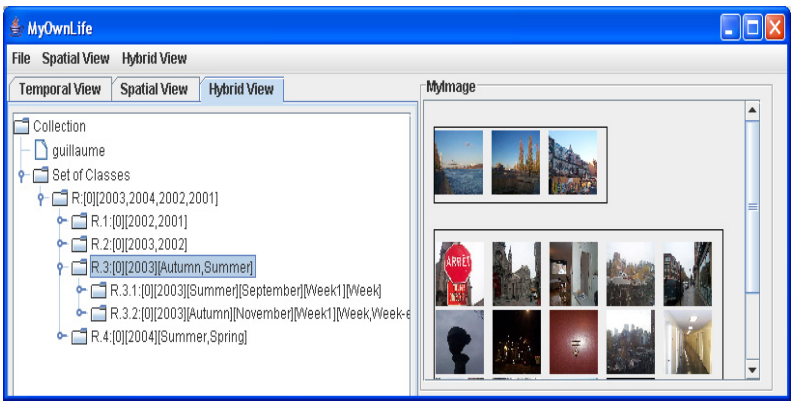

Fig. 4. Screen capture of our prototype MyOwnLife. Here, the hybrid view is selected and the summary of the selected node in the tree is displayed: this node contains 2 events, each one represented by 2 images per each leave included in their subtree (thus, each event of the finer partition appears). 
Finally, we propose a method to provide a hybrid partition of the image collection from the hierarchical temporal classification. It follows the assumption that successive temporal events in a same location are generally connected. Roughly, the approach consists in merging continuous temporal nodes with similar geographical meta-data. Let $q$ the root of the tree, our algorithm proceeds as follows:

1. get back the geographical level summaries of $q$;

2. for each continuous temporal classes $i$ and $j$ (children of $q$ ) with a similar geographical summaries: if $i$ is a leaf and $j$ a node (resp. $j$ is a leaf and $i$ a node) then move $i$ as a new child of $j$ (resp. $j$ as a new child of $i$ ) else merge $i$ and $j$ (a new node containing the children of $i$ and $j$ ).

3. apply step 2 to each child of $q$.

Practically, the obtained hybrid tree presents the property that each node on a same level are temporally and geographically disconnected: the gap between node is due to both a temporal and a location change. Figure 3 presents a combination example of temporal and geographical views.

\section{Experiments and conclusion}

Experiments were carried out on four real user collections: G.B. (721 images taken over 4 years), C.C. (1731 images taken over 3 years) and S.P. (706 images taken over 4 years). All these collections contain images taken on several continents (Asia, North America and Europe). For each collection, we built our hierarchical temporal classification, ask each user to annotate their obtained temporal events to build a geographical decision tree and finally build the geo-temporal view based on the two previous classifications. $T_{\text {diff }}$ and $N_{\text {near }}$ are set respectively to 3 hours and 2 nodes.

The temporal trees obtained for the collection of G.B., C.C. and S.P. are respectively composed of 5, 3 and 3 levels and are well-balanced. The number of children per node varies from 2 to 23 . We notice that our classification extends in depth and width as new data are added. Only a minority of images implies serious restructuring of the tree, and hence the overall computational cost grows almost linearly with the number of images.

\begin{tabular}{|c||c|c|c|}
\hline & Bad & Average & Good \\
\hline \hline Events/Leaves & $0 \%$ & $8 \%$ & $92 \%$ \\
\hline Temporal Summaries & $15 \%$ & $24 \%$ & $61 \%$ \\
\hline Geo-temporal summaries & $0 \%$ & $12.5 \%$ & $87 \%$ \\
\hline \hline Events/Leaves & $5 \%$ & $9 \%$ & $86 \%$ \\
\hline Temporal Summaries & $10 \%$ & $40 \%$ & $50 \%$ \\
\hline Geo-temporal summaries & $6 \%$ & $41 \%$ & $53 \%$ \\
\hline \hline Events/Leaves & $9 \%$ & $10 \%$ & $81 \%$ \\
\hline Temporal Summaries & $5 \%$ & $17 \%$ & $78 \%$ \\
\hline Geo-temporal summaries & $3 \%$ & $8 \%$ & $89 \%$ \\
\hline
\end{tabular}

Table 2. Assessment of the events and summaries respectively for the S.P., C.C. and G.B. collections.
Our prototype MyOwnLife (see fig. 4) was then used to evaluate the obtained partitions. The prototype enables users to browse the different views (temporal, geographical or geotemporal). The panel size containing images is similar to an Iphone screen : figure 4 is then a good example of how our proposal could enhance the browsing task on a mobile device: the events are clearly depicted and summarized with a limited number of images. Another solution could consist in mixing images and textual summaries.

To assess the improvement of the geo-temporal classification, we ask the users to give a mark to both events (leaves of the tree) and summaries (nodes of the tree). Results are reported in the Table 2. For each collection, we obtain a high score for the events, due the small value of $T_{\text {diff }}: 81 \%-$ $92 \%$ of the leaves are considered as Good. The comparison between the temporal and geo-temporal tree shows that our combination of the temporal partition with the geographical textual summaries improves significantly the summaries (an average increase of $13.5 \%$ for the Good summaries). The Bad summaries are due to over-segmentations of connected events (connected events of some summaries are not regrouped in a same node) or under-segmentations (disconnected events in a same node).

This paper deals with the management of personal image collection on mobile devices. Our proposal is an algorithm to build a hierarchical and incremental classification, combining then with geographical meta-data, to provide a geo-temporal partition. We are currently examining solutions for a semisupervised algorithm, taking into account user constraints on the structuring process.

\section{References}

[1] A. Pigeau and M. Gelgon, "Building and tracking hierarchical geographical \& temporal partitions for image collection management on mobile devices," in Proc. of Inter. Conf. of ACM Multimedia, Singapore, Singapore, Nov. 2005, pp. 141-150.

[2] A. Graham, H. Garcia-Molina, A. Paepcke, and T. Winograd, "Time as essence for photo browsing through personal digital libraries," in ACM Joint Conf. on Digital Libraries JCDL, Jun. 2002, pp. 326-335.

[3] J. C. Platt and B. A. Field M. Czerwinski, "PhotoTOC: Automatic clustering for browsing personal photographs," Tech. Rep. MSR-TR-2002-17, Microsoft Research, Feb. 2002.

[4] M. Naaman, Y. J. Song, A. Paepcke, and H. GarciaMolina, "Automatic organization for digital photographs with geographic coordinates," in Proc.of ACM/IEEE Conf. on Digital libraries (JCDL), Jun. 2004, pp. 53-62.

[5] C. Fraley, "Algorithms for model-based Gaussian hierarchical clustering," SIAM Journal on Scientific Computing, vol. 20, no. 1, pp. 270-281, 1999.

[6] C. Biernacki, G. Celeux, and G. Govaert, "Assessing a mixture model for clustering with the integrated classification likelihood," in IEEE Trans. on pattern analysis and machine intelligence, Jul. 2000, vol. 22, pp. 719-725. 